\title{
Spectroscopic Studies of Solvated Hydrogen and Hydroxide Ions at Aqueous Surfaces
}

\author{
Teresa L. Tarbuck, Stephanie T. Ota, and Geraldine L. Richmond*
}

Materials Science Institute and Department of Chemistry, University of Oregon, Eugene, Oregon 97403

*Author to whom correspondence should be addressed. E-mail: Richmond@ oregon.uoregon.edu

\section{Supporting Information}

The neat vapor/water spectral assignments used in this study are described below. One main feature is presented for each mode, although several types of $\mathrm{OH}$ stretching environments comprise each mode. (1) The unbound $\mathrm{OH}$ oscillator (free $\mathrm{OH}$ ) that protrudes into the vapor phase and gives rise to the sharp, distinct feature at $\sim 3700 \mathrm{~cm}^{-1}$. (2) Its companion $\mathrm{OH}$ mode that points toward the bulk liquid phase, which we refer to for simplicity as the donor $\mathrm{OH}$ mode, gives rise to a broad feature at $\sim 3460$ $\mathrm{cm}^{-1}$. Together, these free $\mathrm{OH}$ and donor $\mathrm{OH}$ modes represent more than $20 \%$ of the surface water molecules. ${ }^{1}$ MD calculations by $\mathrm{Buch}^{2}$ and from our group ${ }^{3}$ indicate that the dominant contribution to this response is the donor $\mathrm{OH}$ of these water molecules that interact weakly with neighboring water molecules as a proton and single electron pair donor (the oxygen and the hydrogen both participate in hydrogen-bonding). The highly oriented nature of this doubly bonded donor species leads to its strong VSF contribution. ${ }^{3}$ The frequency of this companion donor $\mathrm{OH}$ mode is similar to that found for the $\mathrm{OH}$ of uncoupled HOD in liquid water. ${ }^{4,5} \mathrm{OH}$ stretching from highly coordinated but more loosely coupled water molecules also make a contribution to this stretching region. (3) Weakly hydrogen bonded water molecules near the interfacial plane give rise to the third type of $\mathrm{OH}$ stretching. These water molecules are most apparent in the sps-polarization spectrum giving rise to a broad feature near 
$3580 \mathrm{~cm}^{-1}$. On the basis of MD simulations by Buch, ${ }^{2}$ and in agreement with our calculations, ${ }^{3}$ the water molecules are nearly parallel to the interface, reside in the topmost layer, and form one to three hydrogen bonds with other water molecules in a loosely coupled geometry. Water molecules solvating surface ions are also seen in this high-energy region. ${ }^{6-8}$ (4) Strongly coordinated water molecules, including tetrahedrally coordinated water in a variety of environments and with a variety of hydrogen-bonding strengths, contribute to the intensity below $3500 \mathrm{~cm}^{-1}$, and generally reside a few angstroms from the top layer. They have a higher average number of bonds per molecule and stronger bond geometry than water molecules in the top surface layer, and can interact cooperatively with the extended water network. ${ }^{2,9}$ A small population of triply bonded companion donor species are found in this spectral region and interfacial depth. ${ }^{2,3}$ For simplicity, this region is referred to as the strongly coupled region or tetrahedrally bonded region due to the large presence of such species in this spectral range. The collective $\mathrm{OH}$ stretching of these highly coordinated water molecules occurs at 3330 and $3200 \mathrm{~cm}^{-1}$. The intensity at $3200 \mathrm{~cm}^{-1}$ (from water molecules with these strong hydrogen bonds) is relatively small. However, the orientation and bonding of these stronger hydrogen-bonded water molecules are very sensitive to surface dipole and electric field effects. These effects can extend the surface region further into the bulk allowing more highly coordinated water molecules to contribute to the VSFS response.

A summary of the fitting parameters for $\mathrm{H}^{+}, \mathrm{OH}^{-}$, and $\mathrm{H}_{2} \mathrm{O}$ are presented in Table 1.

Table 1: Fitting Parameters for the Resonant Peaks

\begin{tabular}{|c|c|c|c|c|c|c|c|c|c|c|}
\hline & \multicolumn{2}{|c|}{ Strong Coord. } & \multicolumn{2}{|c|}{ Strong Coord. } & \multicolumn{2}{|c|}{$*$ Donor $\mathrm{OH}$} & \multicolumn{2}{|c|}{ Free $\mathrm{OH}$} & \multicolumn{2}{|c|}{ Solvation } \\
\hline & $\omega_{v}$ & $\Gamma_{v}$ & $\omega_{v}$ & $\Gamma_{\mathrm{v}}$ & $\omega_{v}$ & $\Gamma_{v}$ & $\omega_{v}$ & $\Gamma_{v}$ & $\omega_{v}$ & $\Gamma_{v}$ \\
\hline $\mathrm{H}_{2} \mathrm{O}$ & 3200 & 45 & 3330 & 130 & 3458 & 90 & 3700 & 18 & & \\
\hline $\mathrm{H}^{+}$ & 3200 & 65 & 3330 & 120 & 3453 & 90 & 3700 & 18 & 3525 & 60 \\
\hline $\mathrm{OH}^{-}$ & 3200 & 55 & 3330 & 150 & 3450 & 90 & 3700 & 18 & 3635 & 60 \\
\hline
\end{tabular}




\section{References}

1. $\quad$ Du, Q.; Superfine, R.; Freysz, E.; Shen, Y. R. Phys. Rev. Lett. 1993, 70, 2313-2316.

2. $\quad$ Buch, V. J. Phys. Chem. B 2005, 109, 17771-17774.

3. $\quad$ Walker, D.; Richmond, G. L. Submitted to J. Phys. Chem. B 2006.

4. $\quad$ Wall, T. T.; Horning, D. F. J. Chem. Phys. 1965, 43, (6), 2079-2087.

5. $\quad$ Scherer, J. R.; Go, M. K.; Kint, S. J. Phys. Chem. 1974, 78, (13), 1304-1313.

6. Raymond, E. A.; Richmond, G. L. J. Phys. Chem. B 2004, 108, 5051-5059.

7. $\quad$ Strauss, I. M.; Symons, M. C. R. J. Chem. Soc., Faraday Trans. 1978, 1, 2518-2529.

8. Walrafen, G. E. J. Chem. Phys. 1971, 55, (2), 768-792.

9. Raymond, E. A.; Tarbuck, T. L.; Brown, M. G.; Richmond, G. L. J. Phys. Chem. B 2003, 107, 546-556. 